\title{
Efficient Algorithm for Linear Pattern Separation
}

\author{
Claude Tadonki ${ }^{1}$ and Jean-Philippe Vial ${ }^{2}$ \\ 1 University of Geneva, Centre Universitaire d'Informatique \\ 24, rue Général Dufour, 1211 Genève 4 - Switzerland \\ claude.tadonki@cui.unige.ch \\ 2 HEC/LOGILAB, 40 Bd du Pont d'Arve, CH-1211 Geneva - Switzerland \\ jean-philippe.vial@hec.unige.ch
}

\begin{abstract}
We propose cutting plane algorithm for solving the linear pattern separation problem, which is a particular case of the more general topic of data mining. The solution we provided, based on convex programming, can also be applied to any other pattern separation scheme based on a convex discriminant like linear piecewise or quadratic models. Some experimentations are reported with different large databases together with a comparison with a direct implemention with two commercial specialized codes.
\end{abstract}

\section{Introduction}

Linear separation [15] is an important concept in data mining [36]. It is widely used and has been applied in many fields, e.g., cancer diagnosis, human genome , game strategies, pattern recognition, decision/selection making, and others. Many other separation rules can be found in the litterature, and our method can handle those of them that are based on a functional rule expressed by a convex form. In some cases the size of the data set [2] is so large that solving the mathematical programming problem becomes a challenge even with the stateof-the-art optimization software. In this paper we propose to resort a so-called cutting plane method to solve the problem efficiently, and we discuss ways to improve performance on the linear separation problem.

\section{Problem Formulation}

Given a set of points $\mathcal{A}=\left\{a_{i} \in \mathcal{R}^{n}, i=1,2, \cdots, N\right\}$, and a partition $\mathcal{S}_{1} \cup \mathcal{S}_{2}$ of the set of indices $\mathcal{S}=\{1,2, \cdots, N\}$, we wish to find $w \in \mathcal{R}^{n}$ and $\gamma \in \mathcal{R}$ such that the hyperplane $\left\{x \mid w^{T} x=\gamma\right\}$ separates the two subsets $\mathcal{A}\left(\mathcal{S}_{1}\right)$ and $\mathcal{A}\left(\mathcal{S}_{2}\right)$, where

$$
\begin{aligned}
& \mathcal{A}\left(\mathcal{S}_{1}\right)=\left\{a_{i} \in \mathcal{A} \mid i \in \mathcal{S}_{1}\right\}, \\
& \mathcal{A}\left(\mathcal{S}_{2}\right)=\left\{a_{i} \in \mathcal{A} \mid i \in \mathcal{S}_{2}\right\} .
\end{aligned}
$$


For typographical convenience, we will write $(w, \gamma)$ instead of $\left(w^{T}, \gamma\right)$.

Actually, one looks for a strong separation. Thus, given a separation margin $\nu>0$, we hope to achieve the separation properties (3.4) displayed bellow

$$
\begin{array}{ll}
\forall a_{i} \in \mathcal{A}\left(\mathcal{S}_{1}\right) & w^{T} a_{i} \geq \gamma+\nu, \\
\forall a_{i} \in \mathcal{A}\left(\mathcal{S}_{2}\right) & w^{T} a_{i} \leq \gamma-\nu .
\end{array}
$$

In general, there is no guarantee that the two sets can be strongly separated. Therefore, for any choice of $w$ and $\gamma$, we might observe misclassification errors, which we define as follows

$$
\begin{array}{cl}
e_{i}^{1}=\frac{\max \left(-w^{T} a_{i}+\gamma+\nu, 0\right)}{\|(w, \gamma, \nu)\|}, & i \in \mathcal{S}_{1}, \\
e_{i}^{2}=\frac{\max \left(w^{T} a_{i}-\gamma+\nu, 0\right)}{\|(w, \gamma, \nu)\|}, & i \in \mathcal{S}_{2} .
\end{array}
$$

Our goal is then to build a separation hyperplane $\left\{x \mid w^{T} x=\gamma\right\}$ (i.e., compute $w$ and $\gamma$ ) for which the total sum of misclassification errors is minimal. In other words, we want to find a vector $w$ and a scalar $\gamma$ such that the average sum of misclassifications errors is minimized [5].

The separation margin $\nu$ helps avoiding the useless trivial solution $(w, \gamma)=$ $(0,0)$. Its value is usually set to 1 . In some cases the separation margin may lead to large values for $w$ and $\gamma$. It may be necessary [2] to bound $w$ to avoid this undesirable feature; so, we add the constraint $\|w\|_{2} \leq k$.

Formally, we have to solve the following optimization problem

$$
\begin{aligned}
\min _{(w, \gamma) \in \mathcal{R}^{n} \times \mathcal{R}}\left[\frac{1}{\left|\mathcal{S}_{1}\right|} \sum_{i \in \mathcal{S}_{1}} \max \left(-w^{T} a_{i}+\gamma+\nu, 0\right)+\right. \\
\left.\frac{1}{\left|\mathcal{S}_{2}\right|} \sum_{i \in \mathcal{S}_{2}} \max \left(w^{T} a_{i}-\gamma+\nu, 0\right)\right]
\end{aligned}
$$

subject to: $\|w\|_{2} \leq k$.

In ACCPM the square normed in the constraint $\|w\|^{2} \leq k^{2}$ is also treated as black box. If $\bar{w}$ is not feasible $\left(\|\bar{w}\|^{2}>k^{2}\right)$, the constraint

$$
w+2\left\langle{ }^{-}, w-\bar{w}\right\rangle \leq k^{2}
$$

holds for any feasible point.

Finally, let us give two bounds on $f$. Since $f(0,0)=2 \nu$, then $2 \nu$ is an upper bound of the optimal value of the objective. A straightforward lower bound is 0 , but this can be only attained if perfect classification is achieved.

Let us discuss now the formulation of problem (7)-(8) as a standard mathematical programming problem. Let $z_{i}, i \in \mathcal{S}$. be an auxiliary variable. The original problem becomes

$$
\min _{\substack{(w, \gamma) \in \mathcal{R}^{n} \times \mathcal{R} \\ z \geq 0}} \frac{1}{\left|\mathcal{S}_{1}\right|} \sum_{i \in \mathcal{S}_{1}} z_{i}+\frac{1}{\left|\mathcal{S}_{2}\right|} \sum_{i \in \mathcal{S}_{2}} z_{i}
$$




$$
\begin{array}{ll}
\text { subject to: } & z_{i} \geq\left(-w^{T} a_{i}+\gamma+\nu\right), i \in \mathcal{S}_{1} \\
& z_{i} \geq\left(w^{T} a_{i}-\gamma+\nu\right), i \in \mathcal{S}_{2} \\
& \|w\|_{2} \leq k .
\end{array}
$$

Note that the constraints (11)-(12) are numerous but linear. The problem is thus a large linear programming problem with one quadratic constraint (13). Some authors 2 prefer to replace the quadratic constraint by a quadratic penalty term in the objective. Another possibility consists in replacing the Euclidean norm in (13) by the $\ell_{\infty}$ norm [4]. The problem becomes then fully linear.

Table 1. Comparison with direct methods

\begin{tabular}{|r|r||r|r||r|r||r|}
\hline$n$ & $m$ & MOSEK $(1)$ & CPLEX(1) & MOSEK $(2)$ & CPLEX $(2)$ & ACCPM \\
\hline \hline 10 & 10000 & 2.54 & 1.95 & 6.31 & 1.81 & 1.95 \\
\hline 20 & 10000 & 2.21 & 2.47 & 11.37 & 2.35 & 2.63 \\
\hline 30 & 10000 & 4.45 & 4.45 & 18.70 & 4.12 & 3.40 \\
\hline 40 & 10000 & 6.34 & 6.11 & 23.78 & 5.99 & 4.50 \\
\hline 50 & 10000 & 9.23 & 8.18 & 26.11 & 8.20 & 4.95 \\
\hline 60 & 10000 & 11.84 & 11.10 & 30.78 & 11.10 & 6.30 \\
\hline 70 & 10000 & 15.13 & 13.07 & 40.87 & 12.82 & 8.86 \\
\hline 80 & 10000 & 19.87 & 15.00 & 50.21 & 14.21 & 10.16 \\
\hline 90 & 10000 & 26.04 & 19.97 & 69.20 & 19.46 & 15.03 \\
\hline 100 & 10000 & 30.22 & 22.19 & 62.63 & 21.29 & 16.81 \\
\hline 10 & 100000 & 143.86 & 81.08 & 113.40 & 78.12 & 5.11 \\
\hline 20 & 100000 & 120.47 & 109.53 & 132.25 & 108.29 & 8.22 \\
\hline 30 & 100000 & 172.21 & 143.98 & 179.24 & 141.31 & 10.59 \\
\hline 40 & 100000 & 253.38 & 194.34 & 215.89 & 190.40 & 16.31 \\
\hline 50 & 100000 & 311.35 & 223.71 & 280.87 & 219.60 & 16.95 \\
\hline 60 & 100000 & 576.77 & 273.46 & 303.09 & 285.38 & 18.97 \\
\hline 70 & 100000 & 742.84 & 408.01 & 411.66 & 396.50 & 28.88 \\
\hline 80 & 100000 & 850.15 & 427.15 & 478.61 & 406.19 & 31.25 \\
\hline 90 & 100000 & 906.57 & 496.57 & 590.95 & 439.29 & 34.06 \\
\hline 100 & 100000 & 1443.25 & 543.25 & 680.81 & 493.78 & 40.30 \\
\hline
\end{tabular}

(1) simplex

(2) interior point

\section{Implementation}

In our experiments we have considered various sample data sets to test our algorithm, all generated radonmly using a normal distribution.

The problem were solved using the public release of ACCPM. All our experiments were conducted on a $500 \mathrm{Mhz}$ SUN Ultrasparc with $256 \mathrm{MB}$ of RAM. For problems with a very large data set, we performed an out-of-core computation. With a buffer of 200, 000 elements, five accesses to disk per iteration were required. 
We compare our method with direct methods based on a standard linear programming (LP) formulation of the problem. Recall that the linear programming formulation was given in (10)-(12). (Note that we drooped the norm constraint (13).) The (dual) LP formulation can be solved using standard techniques of linear programming such as simplex or interior point methods. We have compared ACCPM with two linear programming codes: MOSEK and CPLEX. Both offer the options between a simplex and a primal-dual log barrier algorithm. Table 1 displays our experimental results.

\section{Conclusion}

We have proposed a cutting plane algorithm for the linear pattern separation problem. The main idea was to modeling the problem through the purpose of minimizing the total misclassification gap as a convex function. Experimentations and comparative study show that our method is quiet efficient even when consider very large databases.

Acknowledgement. The authors thank Olivier Péton and Cesar Beltran for their useful comments.

\section{References}

1. R.A. Bosh and J.A. Smith, Separating Hyperplanes and the Authorship of the Disputed Federalist Papers, American Mathematical Monthly, Volume 105, No 7, pp. 601-608, 1995.

2. M.C. Ferris and T.S. Munson, Interior Point Methods for Massive Support Vector Machines, Cours/Sèminaire du $3^{e}$ cycle romand de recherche opèrationnelle, Zinal, Switzerland, march 2001.

3. J. Han and M. Kamber, Data Mining: Concept and Techniques, Morgan Kaufmann Publishers, 2000.

4. O.L. Mangasarian, Linear and Non-linear Separation of Patterns by linear programming, Operations Research, 13, pp. 444-452.

5. O.L. Mangasarian, R. Setino, and W. Wolberg, Pattern Recognition via linear programming: Theory and Applications to Medical Diagnosis, 1990.

6. M.S. Viveros, J.P. Nearhos, M.J. Rothman, Applying Data Mining Techniques to a Health Insurance Information System, 22nd VLDB Conference, Mumbai (Bombay), India, 1996, pp. 286-294. 